\title{
Impact of the COVID-19 pandemic on the management of cancer patients: the experience of the cancer outpatients department of a university hospital in Paris
}

\author{
Authors: Camille Tlemsani, ${ }^{\mathrm{A}}$ Jennifer Arrondeau, ${ }^{\mathrm{B}}$ Sixtine De Percin, ${ }^{\mathrm{B}}$ Ithar Gataa, ${ }^{\mathrm{B}}$ Marie Bretagne, ${ }^{\mathrm{C}}$ Zahra Ajgal, ${ }^{\mathrm{C}}$ \\ Olivier Huillard, ${ }^{\mathrm{B}}$ Marie Wislez, ${ }^{\mathrm{D}}$ Romain Coriat, ${ }^{\mathrm{D}}$ Jerome Alexandre, ${ }^{\mathrm{D}}$ Pascaline Boudou-Rouquette ${ }^{\mathrm{B}}$ and \\ François Goldwasser ${ }^{\mathrm{D}}$
}

\begin{abstract}
Cancer patients are a highly vulnerable group in the COVID-19 pandemic and it has been necessary for oncology units to adapt to this unexpected situation. We present our management of outpatients with cancer during the pandemic. We applied two major adaptations: extending the intervals between injections for maintenance therapy and protocol adaptation for patients with comorbidities. Between 17 March and 30 April 2020, 406 patients were treated in our outpatients department. Protocols were adapted for 94 (23.1\%) patients. Among them, $49 \%$ had an extended interval between treatment administrations, $22.3 \%$ had modified protocols to reduce toxicity, $20.2 \%$ had therapeutic interruptions and $5.3 \%$ did not receive their treatment because of a COVID-19 infection. Overall, protocol adaptations concerned more than $20 \%$ of the patients. This pandemic was an opportunity for oncologists to re-examine the risk versus benefit balance of administering immunosuppressive treatment and highlighted that oncology daily routine should not be applied automatically.
\end{abstract}

KEYWORDS: COVID-19, pandemic, outpatients, cancer, protocol adaptations, comorbidities, maintenance therapy

DOI: 10.7861/clinmed.2020-0666

\section{Introduction}

In France, one of the countries mostly affected by the COVID-19 pandemic, confinement measures for the whole population started on 17 March 2020.

The first Chinese nationwide study suggested that cancer patients had a higher risk of COVID-19 infection ( $1 \%$ vs $0.29 \%$ )

Authors: Amaître de conférences des universités - praticien hospitalier, Cochin Hospital, Paris, France; Bpraticien hospitalier, Cochin Hospital, Paris, France; ${ }^{C}$ Chef de clinique assistant, Cochin Hospital, Paris, France; Dprofesseur des universités - praticien hospitalier, Cochin Hospital, Paris, France and a higher risk of need for intensive care assistance (39\% vs $8 \% ; p=0.0003) .{ }^{1}$ Hospital admission and recurrent hospital visits were found to be a potential risk factor for COVID-19 infection. ${ }^{2}$ A history of cancer treatment or surgery in the past month was also associated with poor prognosis. ${ }^{2}$ These data have been confirmed in another study showing that injection of an antitumour treatment within 14 days of the COVID-19 diagnosis significantly increased the risk of developing severe events (hazard ratio $[H R] 4.079,95 \%$ CI 1.086-15.322, $p=0.037) .{ }^{3}$ Moreover, recent data from the global registry TERAVOLT ('Thoracic cancERs international coVID 19 cOLlaboraTion') showed that the presence of chronic obstructive pulmonary disease (COPD) was associated with an increased risk of hospitalisation in univariate analysis, and that the presence of more than one comorbidity was associated with an increased risk of hospitalisation and death in lung cancer patients. ${ }^{4}$ Overall, cancer patients seem at a higher risk of severe events in $48-54 \%$ of cases and of death in $5.6-29 \%$ cases. ${ }^{1,3}$

At the request of the French Health Ministry, French national recommendations for cancer patient management have been published on 25 March 2020. ${ }^{5}$ These guidelines stressed the necessity to prioritise patients on a case-by-case basis, prioritising curative rather than palliative therapy, patients aged under 60 or with life expectancy of 5 years or more, and patients in whom tumour lesion extension or symptoms might jeopardise their lives quickly in the case of treatment discontinuation. ${ }^{5}$

Oncology units had to adapt in a hurry to this unexpected pandemic and difficult decisions about how and when to provide cancer treatment had to be made. We present how our institute (Cochin Hospital, University Hospital in Paris) managed cancer patients during the COVID-19 pandemic.

\section{Methods}

Patients were screened for the presence of COVID-19 symptoms by a phone call from a nurse the day before the visit to the hospital. COVID-19 symptoms were defined as fever, cough, rhinorrhea, myalgias, anosmia, taste loss, headache, confusion and increasing asthenia. Visitors were not allowed to come into the hospital, and we mandated mask protection for both the patient and the staff and conducted temperature checks before entrance into the department. Consistent with the French national recommendations, we applied two major actions: to extend the injection intervals for patients 

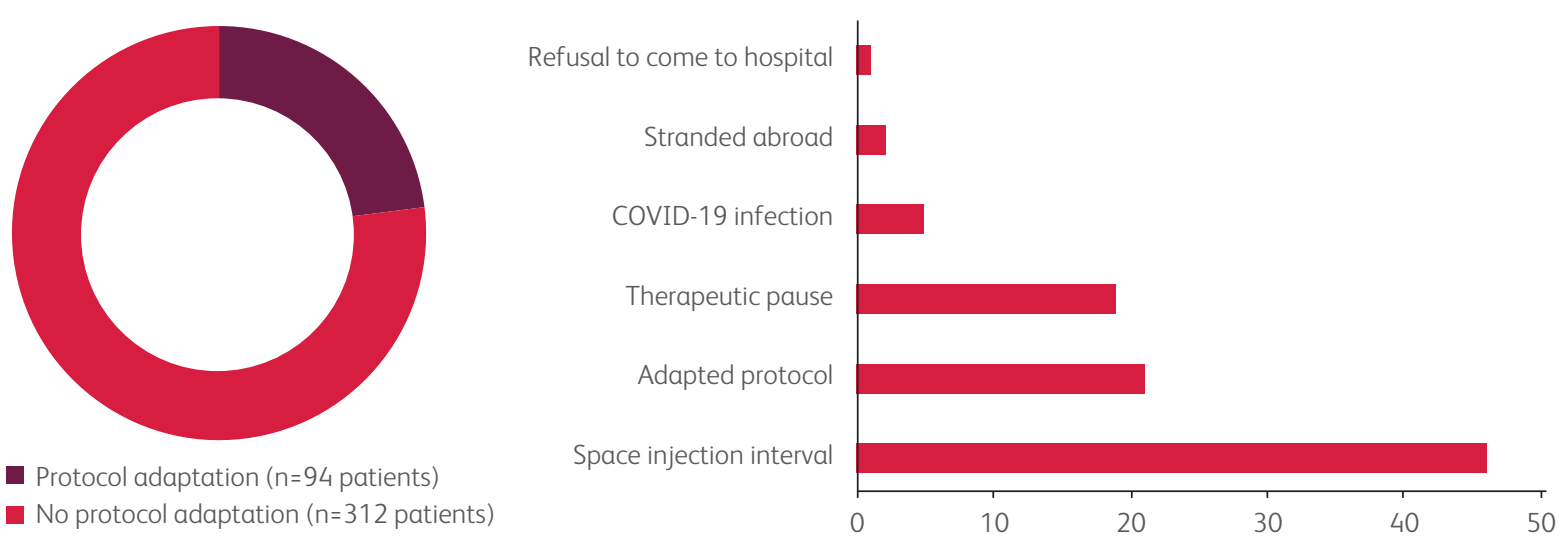

Fig 1. Summary of the cancer patient's management in the inpatient unit. a) The proportion of patients who had a cancer treatment protocol adaptation (dark red) and the proportion of patients with no cancer treatment protocol adaptation (red). b) The number of patients experiencing each type of protocol adaptation.

receiving systemic maintenance therapy with immunotherapies or antibodies, and to adapt protocols for elderly patients or patients with comorbidities after a collective discussion between the oncologist of the patient and the oncologists in the unit.

This study was approved by the institutional review board according to good clinical practice, applicable laws, and the declaration of Helsinki. The number reference is AJ20-75032.

\section{Results}

Between 17 March and 30 April 2020, 406 patients came to the oncology outpatients department, representing 815 hospitalisations. Among them, 116 (28.6\%) had a gastrointestinal cancer, 112 (27.6\%) had a lung cancer, 85 (20.9\%) had a gynaecological cancer, 64 (15.8\%) had a urological cancer, 22 (5.4\%) had a sarcoma, and seven $(1.7 \%)$ had other cancers. At 815 , the number of hospitalisations was $18.4 \%$ lower than the same period in 2019.

Protocol adaptations following COVID-19 pandemic were carried out for 94 (23.1\%) patients. Among these, 46 (49\%) had an extension of the interval between injections in the context of maintenance treatment (immunotherapies, monoclonal antibodies), 21 (22.3\%) patients had an adapted protocol in order to reduce toxicities (lower doses or fewer drugs), 19 (20.2\%) had therapeutic interruption, five $(5.3 \%)$ patients did not receive their treatment because of a COVID-19 infection, two (2.1\%) patients were stranded abroad, and one (1.1\%) patient refused to come to hospital (Fig 1).

Patients who had maintenance treatment spacing were mostly patients with lung cancer $(n=19 / 46,41.3 \%)$ and gynaecological cancer ( $n=15 / 46,32.6 \%$ ). Adaptation of protocols and therapeutic interruption were carried out in 18/41 (43.9\%) patients with gastrointestinal cancer, $10 / 41$ (24.4\%) patients with lung cancer, 5/41 (12.2\%) with sarcoma, 4/41 (9.8\%) with urological cancer, $3 / 41(7.3 \%)$ with gynaecological cancer and 1/41 (2.4\%) patient with a carcinoma of unknown primary. Most of the patients with protocol adaptations were male (the female/male sex ratio was 0.8 in the protocol adaptations group compared to 1 in the noprotocol-adaptation group). On the other hand, the median age was the same in the group of patients with protocol adaptations as in the no-protocol-adaptation group (median age 67 years old).
Among the five (1.2\%) patients with COVID-19 infection, four had lung cancer and one had a leiomyosarcoma. All patients except one had received at least two lines of treatment before the COVID-19 infection. Median duration between the last cancer treatment and the first occurrence of COVID-19 symptoms was 3.5 days (range 1-9 days). Four patients had to be hospitalised because of respiratory symptoms. The median delay between first COVID-19 symptoms and hospitalisations was 4.5 days (range 1-14 days). None of them had to be hospitalised in an intensive care unit. Respiratory assistance by oxygen therapy was needed for the four hospitalised patients. The median hospitalisation duration was 6.5 days (range 5-12 days), and none of them died. The median duration of the anticancer treatment interruption was 27 days (range 10-63 days). All patients resumed their cancer treatment, but for two of them cancer treatment was not resumed until after a longer delay (day 58 and 63 after the first occurrence of COVID-19 symptoms) because of asthenia and worsening pulmonary function. The characteristics of the patients with COVID-19 infection are summarised in Table 1.

\section{Conclusions}

To date, most published studies have focused on mortality and prognostic factors in cancer patients with COVID-19 infection. Little is known about treatment administration modifications and their outcomes. ${ }^{6}$ Our experience highlights that changes in treatment management concerned a high proportion of cancer patients. In our series, five patients had COVID-19 infection, which is a similar rate of COVID-19 infection compared to that seen in other published studies. ${ }^{7.8}$ The most frequent treatment adaptation was spacing out the duration of maintenance treatment (immunotherapies or antibodies). Interestingly, in our unit, physicians estimated that reduced doses, lightened protocols or therapeutic interruption would be less deleterious compared to full protocol in 40/406 (9.8\%) patients in the context of COVID-19 pandemic. This observation raises the question of whether risk/ benefit balance should be challenged more often by physicians outside of the context of COVID-19 pandemic.

The first published studies highlighted that cancer patients had a higher risk of COVID-19 infection, a higher risk of need 


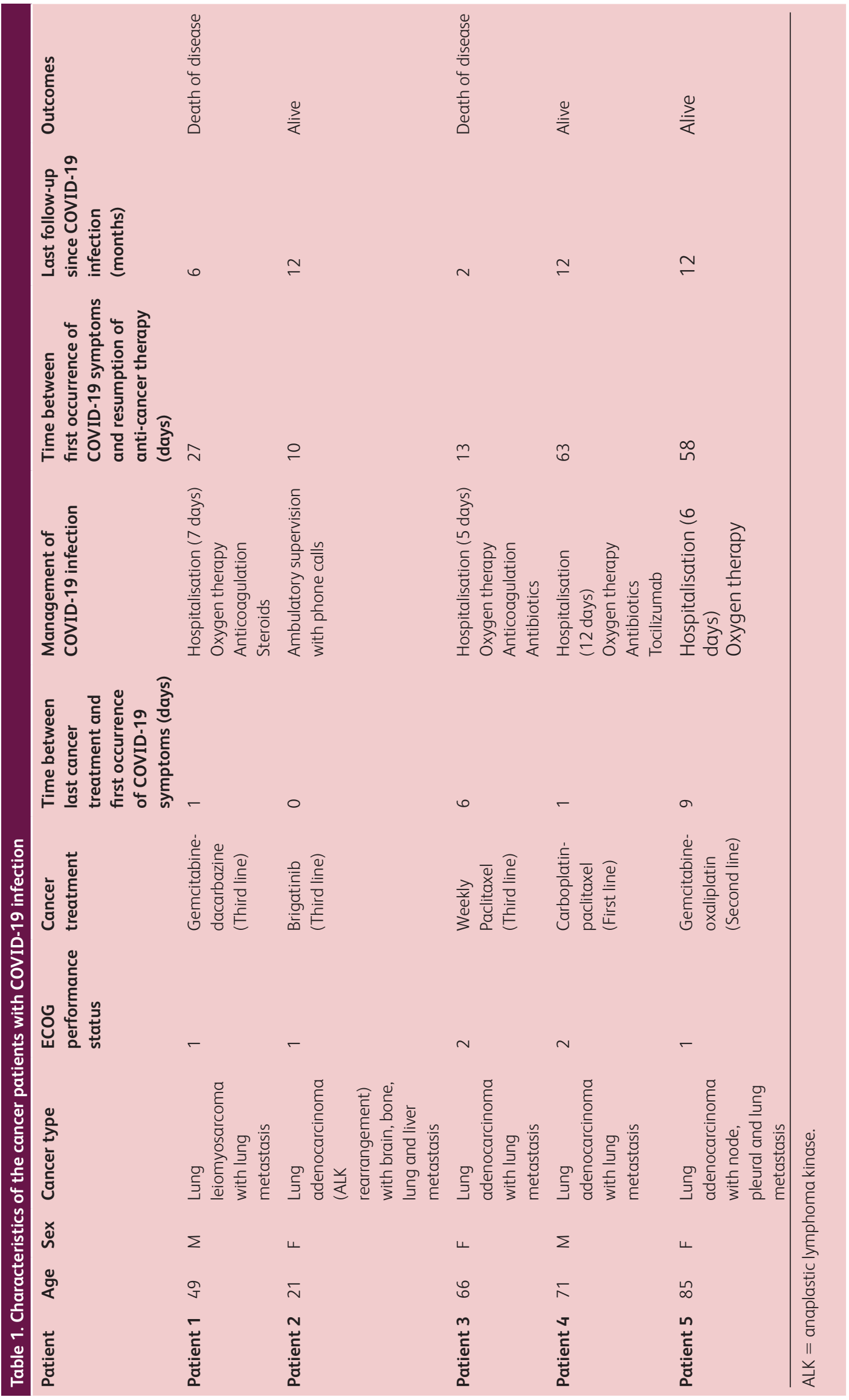


for intensive care assistance and a higher rate of death. ${ }^{1}$ More recently, a prospective study including 800 cancer patients showed that the mortality from COVID-19 in cancer patients appears to be principally driven by age, gender and comorbidities. ${ }^{9}$ Unlike the previous publications, these results suggest that the COVID-19 mortality rate in cancer patients is not so high in comparison with the other patients. Overall, these data suggest that further studies are needed to determine the incidence, prognostic factors and mortality of COVID-19 infection in cancer patients.

In conclusion, this unexpected pandemic required oncology units to quickly adapt the management of cancer patients. In our inpatient unit, more than $20 \%$ patients experienced treatment modification. This pandemic was an opportunity for oncologists to re-examine the risk versus benefit balance of administering immunosuppressive treatments to cancer patients and highlighted that oncology daily routine should be a personalised medicine.

\section{References}

1 Liang W, Guan W, Chen R et al. Cancer patients in SARS-CoV-2 infection: a nationwide analysis in China. Lancet Oncol 2020;21:335-7.

2 Yu J, Ouyang W, Chua MLK, Xie C. SARS-CoV-2 transmission in patients with cancer at a tertiary care hospital in Wuhan, China. JAMA Oncol 2020;6:1108-10.
3 Zhang L, Zhu F, Xie L et al. Clinical characteristics of COVID-19infected cancer patients: a retrospective case study in three hospitals within Wuhan, China. Ann Oncol 2020;31:894-901.

4 Whisenant JG, Trama A, Torri V et al. TERAVOLT: Thoracic cancers international COVID-19 collaboration. Cancer Cell 2020;37:742-5.

5 You B, Ravaud A, Canivet A et al. The official French guidelines to protect patients with cancer against SARS-CoV-2 infection. Lancet Oncol 2020;21:619-21.

6 Waisberg F, Enrico D, Angel M, Chacon M. Cancer treatment adaptations in the COVID-19 era. JCO Oncol Pract 2020;23:16:305-7.

7 Mei H, Dong X, Wang Y, Tang L, Hu Y. Managing patients with cancer during the COVID-19 pandemic: frontline experience From Wuhan. Lancet Oncol 2020;21:634-6.

8 Desai A, Sachdeva S, Parekh T, Desai R. COVID-19 and cancer: lessons from a pooled meta-analysis. JCO Glob Oncol 2020;6:557-9.

9 Lee LY, Cazier JP, Angelis V et al. COVID-19 mortality in patients with cancer on chemotherapy or other anticancer treatments: a prospective cohort study. Lancet 2020;395:1919-26.

Address for correspondence: Dr Camille Tlemsani, Department of Medical Oncology, Cochin Hospital, Assistance PubliqueHôpitaux de Paris (AP-HP), Paris Descartes University, CARPEM, 75014 Paris, France.

Email: camille.tlemsani@aphp.fr 